\title{
Circadian and ultradian cardiovascular rhythmicity in obese children
}

\author{
Christoph Saner $^{1}$ • Giacomo D. Simonetti ${ }^{2,3}$ - Elke Wühl ${ }^{4}$ • Primus E. Mullis ${ }^{5}$. \\ Marco Janner ${ }^{5}$
}

Received: 26 November 2015 /Revised: 21 April 2016 / Accepted: 16 May 2016 /Published online: 30 May 2016

(C) Springer-Verlag Berlin Heidelberg 2016

\begin{abstract}
Altered circadian and ultradian blood pressure (BP) and heart rate (HR) rhythmicity have been described in diseases with increased cardiovascular risk. We analyzed cardiovascular rhythmicity in obese children. BP and HR rhythmicity was assessed with Fourier analysis from 24-h ambulatory BP measurements in 75 obese children and compared with an age- and gender-matched, lean healthy reference group of 150 subjects. Multivariate regression analysis was applied to identify significant independent factors explaining variability of rhythmicity. Prevalence of 24- and 6-h BP rhythmicity in the obese group was lower $(p=0.03$ and $p=0.02)$, whereas the prevalence
\end{abstract}

Communicated by Mario Bianchetti

Electronic supplementary material The online version of this article (doi:10.1007/s00431-016-2736-4) contains supplementary material, which is available to authorized users.

Giacomo D. Simonetti

giacomo.simonetti@eoc.ch

1 University Children's Hospital UKBB, Spitalstrasse 33, CH-4056 Basel, Switzerland

2 Division of Pediatric Nephrology, Department of Pediatrics, Inselspital, Bern University Hospital, University of Bern, Freiburgstrasse 15, CH-3010 Bern, Switzerland

3 Pediatric Department of Southern Switzerland, Via Ospedale, CH-6500 Bellinzona, Switzerland

4 Center for Pediatrics and Adolescent Medicine, University of Heidelberg, Im Neuenheimer Feld 430,

D-69120 Heidelberg, Germany

5 Division of Pediatric Endocrinology, Diabetology and Metabolism, Department of Pediatrics, Inselspital, Bern University Hospital, University of Bern, Freiburgstrasse 15, CH- 3010 Bern, Switzerland of HR rhythmicity was comparable in both groups. Excluding hypertensive participants, the results remained similar. Twenty-four-hour BP and HR acrophase were delayed in obese children $(p=0.004, p<0.0001), 24-\mathrm{h}$ BP amplitude did not differ $(p=0.07)$, and 24-h HR amplitude was blunted $(p=<0.0001)$. BP Mesor in the obese group was higher $(p=0.02)$; HR Mesor did not differ $(p=0.1)$. Multivariate regression analysis failed to identify a single anthropometric or blood pressure parameter explaining the variability of $\mathrm{BP}$ and $\mathrm{HR}$ rhythmicity.

Conclusion: Prevalence and parameters of circadian and ultradian BP and HR rhythmicity in obese children are altered compared to a healthy reference group, independent of preexisting hypertension.

What is Known:

- Altered cardiovascular rhythmicity has been described in children with different diseases such as primary hypertension or chronic renal failure.

What is New:

- This study reveals altered cardiovascular rhythmicity in obese children compared to an age and gender-matched healthy reference group independent from preexisting hypertension.

Keywords Obesity $\cdot$ Rhythmicity $\cdot$ Heart rate $\cdot$ Blood pressure $\cdot$ Hypertension
Abbreviations
ABPM Ambulatory blood pressure measurement
BMI Body mass index
BP Blood pressure
BPM Beats per minute
CRF Chronic renal failure 


$\begin{array}{ll}\text { DBP } & \text { Diastolic blood pressure } \\ \text { GLM } & \text { Generalized linear model } \\ \text { HR } & \text { Heart rate } \\ \text { IQR } & \text { Interquartile range } \\ \text { MAP } & \text { Mean arterial blood pressure } \\ \text { OR } & \text { Odds ratio } \\ \text { PH } & \text { Primary hypertension } \\ \text { SBP } & \text { Systolic blood pressure } \\ \text { SGA } & \text { Small for gestational age } \\ \text { SDS } & \text { Standard deviation score } \\ \text { WCH } & \text { White coat hypertension }\end{array}$

\section{Introduction}

Childhood obesity has reached an epidemic plateau in most industrialized countries [13, 18, 39]. Pathophysiological consequences of obesity on the cardiovascular system regarding structure and function of the vascular system as well as target organ damage are well known [1, 5, 20].

The impact of childhood obesity on individual health is further aggravated with increasing body mass index (BMI) due to associated hypertension [11, 36, 37], diabetes, dyslipidemia, and metabolic syndrome [10, 35]. Since obesity as well as hypertension has been shown to track into adulthood, we are just about to become acquainted with the scale of its sequelae $[12,28]$.

With the implementation of 24-h ambulatory blood pressure measurements (ABPM) for patients at risk, the recorded data lead to a much more precise description of blood pressure and its derived variables. ABPM compared to random blood pressure (BP) measurements or home $\mathrm{BP}$ measurements are more closely correlated with risk factors for future cardiovascular disease [16, 25, 33, 34, 38]. In addition to common parameters, circadian $(24 \mathrm{~h})$ and ultradian rhythm patterns can be assessed, for instance, using Fourier analysis [43].

Circadian rhythms are a cornerstone of physiology and are already present in invertebrates. The central "zeitgeber" of this system in humans is a master clock located in the suprachiasmatic nucleus of the anterior hypothalamus. This master clock coordinates timing of clocks in peripheral organs like the liver, kidneys, or the heart. External signals such as light and feeding fine-tune the master clock to the $24 \mathrm{~h}$ period [29].

Children with chronic renal failure [41], primary hypertension, and white coat hypertension [17] as well as children born small for gestational age [40] have been reported to have different degrees of disruption of cardiovascular rhythmicity. We hypothesized that obese children also could have disturbed cardiovascular rhythmicity and tested for the dependency of $\mathrm{BP}$ values. To test this hypothesis, we compared circadian and ultradian cardiovascular rhythms of a group of obese children to an age and gender-matched lean reference group.

\section{Materials and methods}

\section{Overview}

Obese children aged 4 to 16 years investigated at the University Children's Hospital, Inselspital and University of Bern, Switzerland who had ABPM measurement between January 2011 and December 2013 were analyzed retrospectively. Obesity was defined as BMI $>1.88$ standard deviation according to age- and sex-specific BMI charts using the growth charts of the Swiss Society of Paediatrics [2].

Every single obese subject was compared with two ageand gender-matched lean healthy children, chosen among subjects from a lean reference group described elsewhere [8]. None of the participants was under medical treatment influencing weight, blood pressure, or heart rate.

\section{4-h ambulatory blood pressure measurements}

ABPM was recorded in all individuals on an outpatient basis using an oscillometric device (model 90207 Spacelabs Medical, Issaquah, WA). Diurnal readings were taken every 15-20 min and nocturnal every 30-45 min. The night period was defined from 11:00 PM until 07:00 AM; daytime was set between 07:00 AM until 11:00 PM. ABPM recordings were considered sufficient if $\geq 20$ readings per sampling period and more than 20 continuous hours with valid readings were present as well as sampling gaps were less than $3 \mathrm{~h}$. Hypertension was defined as systolic or diastolic BP $\geq 95$ th percentile for gender and height during $24 \mathrm{~h}$, daytime, or nighttime as defined in the fourth report on diagnosis, evaluation, and treatment of high BP in children and adolescents [22]. We used German reference values provided by the German Working Group on Pediatric Hypertension to classify BP measurements in both cohorts as they are currently considered the best available data set for pediatric ABPM [7, 42].

Further analysis of rhythmicity was restricted to heart rate (HR) and mean arterial BP (MAP) values, as during oscillometric measurements only MAP is measured, whereas systolic and diastolic values are mathematically derived from device-specific algorithms.

Nighttime dipping was analyzed in all subjects calculating the ratio of daytime MAP in relation to nighttime MAP. A lowering of $\geq 10 \%$ MAP was considered as dipping, $<10 \%$ was considered as non-dipping.

\section{Rhythmicity analysis}

ABPM profiles were examined for the prevalence of circadian (24 h) and ultradian (12, 8, 6, 4.8, and $4 \mathrm{~h}$ ) MAP and HR rhythms using Chronos-Fit Software 1.06 [43].

This software combines a partial Fourier analysis with a stepwise regression technique. To simplify, the course of a 


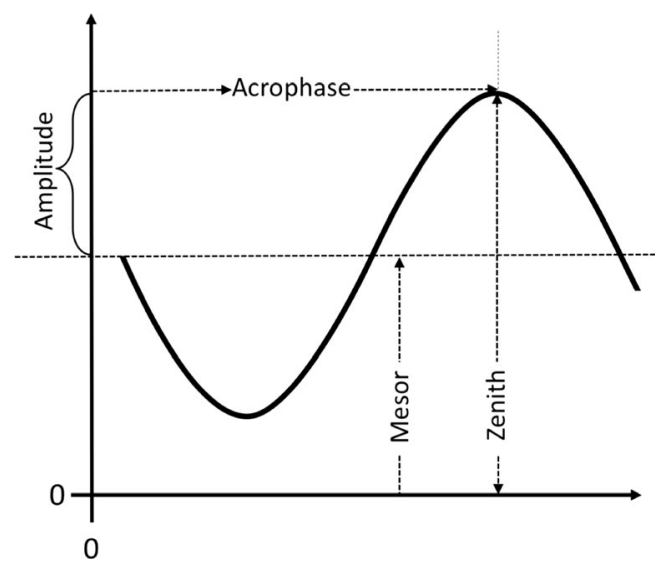

Fig. 1 Terminology of rhythmicity parameters. Cosine wave with any period length and illustration of its derived parameters

24-h MAP or HR profile is described by a wave like function, which results as a summation of simple cosine waves with circadian and ultradian period lengths. For this, circadian and ultradian cosine waves are checked by F-test for its significance to improve the existing model e.g., if the addition of a cosine wave with period length of $6 \mathrm{~h}$ improves the existing model at a level of significance of $p<0.05$, this ultradian rhythm is included into the model. The period length is set for a circadian rhythm at $24 \mathrm{~h}$, for ultradian at $12,8,6,4.8$, and
$4 \mathrm{~h}$. For circadian and ultradian rhythms, the following parameters were calculated (1) the median value midway between the lowest and highest value of the fitted curve (Mesor); (2) the amplitude, which is the difference between Mesor and the highest value; and (3) the acrophase, which is the time from midnight to the zenith of the curve during the rhythm (Fig. 1).

\section{Statistical analysis}

Correlation analyses were performed with the Pearson test for variables with parametric distribution and the Spearman test for variables with non-parametric distribution. The MannWhitney $U$ test was used to compare medians between two groups. Assessment of prevalence for circadian and ultradian MAP and HR rhythms was done using the Chi-square test.

A generalized linear model (GLM) with binomial error distribution and a logit link were used to estimate the contribution of a set of selected variables.

Forward stepwise multivariate linear regression analysis was performed to identify the significant independent factors influencing amplitudes and acrophases of the MAP and HR rhythms.

All statistical analyses were performed using RStudio software, version 0.98.1102 or GraphPad Prism software, version
Table 1 Anthropometric and clinical characteristics of obese and healthy participants

\begin{tabular}{llll}
\hline & Healthy, $n=150$ & Obese, $n=75$ & $p$ value \\
\hline Female (\%) & $68(45.3)$ & $34(45.3)$ & 1 \\
Age (years) & $11.0(8.0-13.0)$ & $11.6(9.0-13.6)$ & 0.22 \\
Weight (kg) & $40.8(29.0-51.3)$ & $71.0(52.3-85.0)$ & $<0.001^{\text {a }}$ \\
Height (cm) & $149.0(133.8-162.0)$ & $156.3(140.1-166.7)$ & $<0.05^{\mathrm{a}}$ \\
Height (SDS) & $0.8(-0.2$ to 1.5$)$ & $1.3(0.7-1.9)$ & $<0.01^{\mathrm{a}}$ \\
BMI (kg/m2) & $17.8(16.2-20.1)$ & $28.1(25.4-31.5)$ & $<0.001^{\mathrm{a}}$ \\
BMI (SDS) & $0.3(-0.4$ to 1.1$)$ & $2.8(2.5-3.4)$ & $<0.001^{\mathrm{a}}$ \\
SBP 24 h SDS & $-0.2(-0.8$ to 0.4$)$ & $-0.2(-1.1$ to 1.0$)$ & 0.57 \\
SBP Day SDS & $-0.2(-0.7$ to 0.3$)$ & $-0.3(-1.1$ to 0.9$)$ & 0.90 \\
SBP Night SDS & $-0.1(-0.8$ to 0.4$)$ & $0.3(-0.3$ to 1.6$)$ & $<0.001$ \\
DBP 24 h SDS & $0.0(-0.8$ to 0.5$)$ & $-0.3(-1.0$ to 0.6$)$ & 0.33 \\
DBP Day SDS 24 h & $-0.1(-0.8$ to 0.6$)$ & $-0.4(-1.0$ to 0.4$)$ & 0.13 \\
DBP Night SDS 24 h & $0.1(-0.7$ to 0.7$)$ & $0.4(0-1.4)$ & $<0.01$ \\
MAP SDS 24 h & $-0.1(-0.7$ to 0.5$)$ & $-0.1(-0.7$ to 0.9$)$ & 0.22 \\
Heart rate 24 h (bpm) & $84(78-90)$ & $86(80-91)$ & 0.20 \\
Heart rate day (bpm) & $90(83-97)$ & $90(83-96)$ & 0.77 \\
Heart rate night (bpm) & $70(65-78)$ & $79(72-84)$ & $<0.001$ \\
Pulse pressure (mmHg) & $42(39-47)$ & $46(41-51)$ & $<0.01$ \\
Dipping $<10 \%$ ( $n)$ & $23(15.3 \%)$ & $30(40 \%)$ & $<0.001^{\mathrm{b}}$ \\
Hypertension $(n)$ & $17(11.3 \%)$ & $23(30.6 \%)$ & $<0.001^{\mathrm{b}}$ \\
\hline
\end{tabular}

Values are given either as median and interquartile range or as relative frequency

${ }^{a}$ Relevant parameters for group allocation

${ }^{\mathrm{b}}$ The $p$ values for the prevalence of dipping and hypertension was calculated using Chi-square test; all other $p$ values are given after performing Mann-Whitney $U$ test 
6.0 (GraphPad Software, San Diego, California, USA). Significance was determined at $p<0.05$.

\section{Patient's medical reports}

\section{Characteristics of the study population}

Seventy-five obese children, $45.3 \%$ girls, with a median age of 11.6 years (interquartile range (IQR) 9.0-13.6) were compared to an age- and gender-matched healthy lean reference group of 150 participants, $45.3 \%$ girls, with a median age of 11.0 years (IQR 8.0-13.0). The anthropometric and blood pressure characteristics of the two groups are shown in Table $1.23(30.6 \%)$ obese participants and $17(11.3 \%)$ participants of the lean reference group showed hypertensive blood pressure values $(p=0.0003)$. Nighttime diastolic and systolic blood pressures (DBP, SBP) were significantly higher in obese children. Furthermore, obese children showed a higher heart rate during the night and had higher 24 -h pulse pressure (Table 1). Finally, the prevalence of nighttime non-dipping in obese children was higher compared to healthy children (30 (40\%) obese children vs. 23 (15.3\%) healthy children, $p<0.0001$ (Table 1)).

\section{Results}

\section{Circadian and ultradian cardiovascular rhythms}

The prevalence of the 24-h mean arterial BP (MAP) rhythm and of the 6-h MAP rhythm was significantly lower in obese children ( $p=0.03$ and $p=0.02$ ), whereas the prevalence of the 12-, 8-, 4.8- and 4-h MAP rhythms were similar between both groups (Fig. 2a).

The prevalence of all ultradian and circadian heart rate (HR) rhythms was comparable between both groups (Fig. 2b).

Excluding hypertensive participants according to the above mentioned definition, the prevalence for the 24- and 6-h MAP rhythms remained significantly lower in obese children $(p=0.02$ and $p=0.03)$, whereas the prevalence for the remaining ultradian rhythms was similar. In addition, the prevalence for the ultradian and circadian HR rhythms between healthy and obese subjects was conserved after exclusion of hypertensive participants.

The MAP Mesor was significantly higher in obese children $(p=0.02)$, but the HR Mesor did not differ significantly between both groups $(p=0.1)$.

Only the 4.8-h MAP amplitude of the obese children was significantly higher compared to the reference group $(p=0.04)$, whereas the MAP amplitudes of $24,12,8,6$, and $4 \mathrm{~h}$ rhythms were similar between obese and healthy participants (Fig. 3a). The 24-h HR amplitude of the
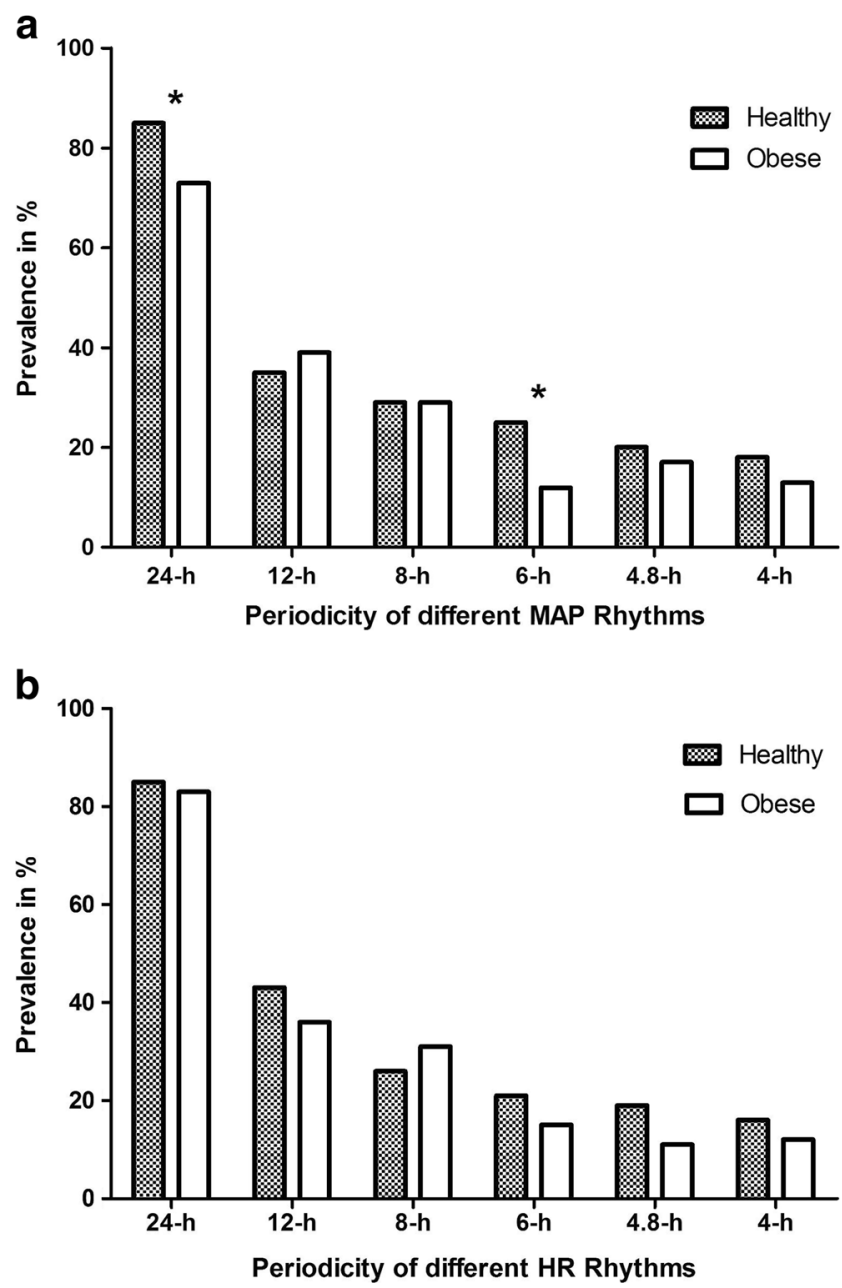

Fig. 2 a Prevalence of MAP rhythms, obese $n=75$, healthy $n=150$, ${ }^{*} p<0.05$ using Chi-square test. b Prevalence of HR rhythms, obese $n=75$, healthy $n=150$

obese cohort was blunted ( $p<0.0001$, Fig. $3 b)$. Both the acrophases of the 24-h MAP rhythm and of the 24-h HR rhythm were delayed in obese children $(p=0.004$ and $p<0.0001$ ), but we observed no difference for the acrophases of the shorter MAP or HR rhythms (Fig. 3c, d).

\section{Associations between cardiovascular rhythmicity and subjects characteristics}

Multivariate analysis included age (years), sex, height standard deviation score (SDS), BMI-SDS, 24-hour MAP SDS, and 24-h HR in beats per minute (bpm) as independent variables and the prevalence of circadian and ultradian rhythms as dependent variables (Table 2). Each increase of age by 1 year, matched for the other included variables, significantly increased the prevalence of the 12-h MAP, 12-h HR, and 6-h HR rhythm (odds ratio (OR) $1.15,1.16$, and 1.22). The prevalence of the 24-h HR rhythm though was decreased (OR 0.86). Increasing 24-h MAP by one SDS significantly increased the prevalence of 4-h MAP rhythm 
(OR 1.52) in boys and girls, whereas among females, the prevalence of the 4-h MAP rhythm (OR 0.35) was decreased. The prevalence of all other circadian and ultradian MAP and HR rhythms could not be significantly explained by any of the included variables.

Multivariate regression analysis failed to identify a single anthropometric or blood pressure parameter

a

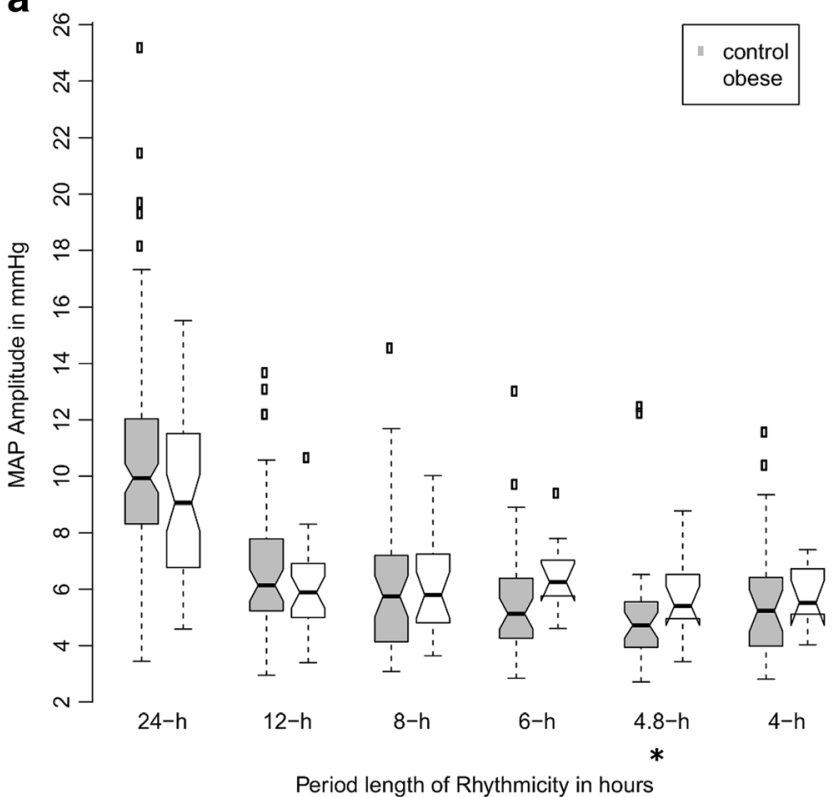

C

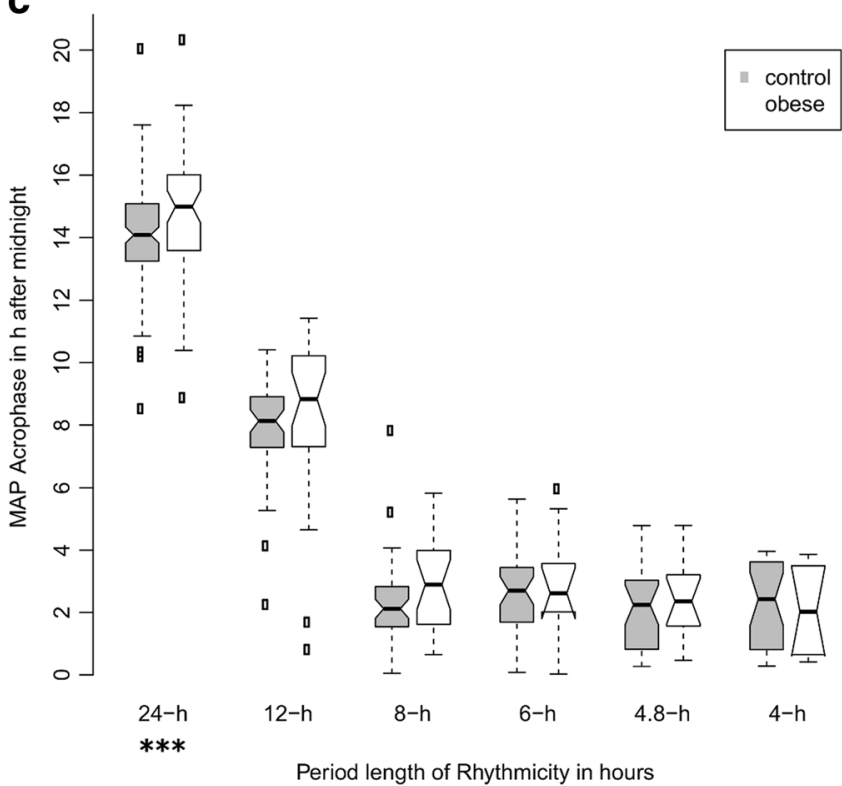

Fig. 3 Notched box plots of MAP and HR amplitudes and acrophases. Notched box plot. The box shows the interquartile range (IQR $=25$ th75 th percentile). The bold crossline indicates the median of the data. The notch indicates the $95 \%$ confidence interval around the median. The whiskers add 1.5 times the IQR to the 75th percentile and subtracts 1.5 times the IQR from the 25th percentile. Outliers above and under the whiskers are shown with small circles. a MAP amplitudes of circadian explaining the variability of $\mathrm{BP}$ and $\mathrm{HR}$ amplitudes and acrophases of all the circadian and ultradian rhythms. The results of the multivariate stepwise regression analysis including age, sex, height-SDS, BMI-SDS, 24-hour MAP-SDS, and 24-h HR (bpm) of obese children only and of obese and lean children together into the model, are shown in the supplementary Tables 1 and 2 .

b

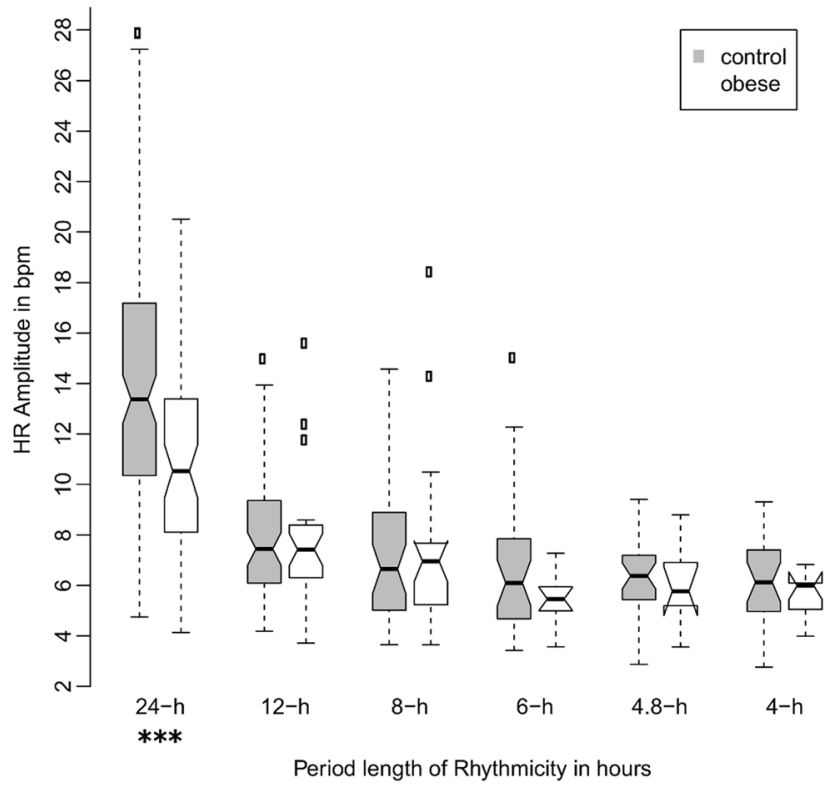

d

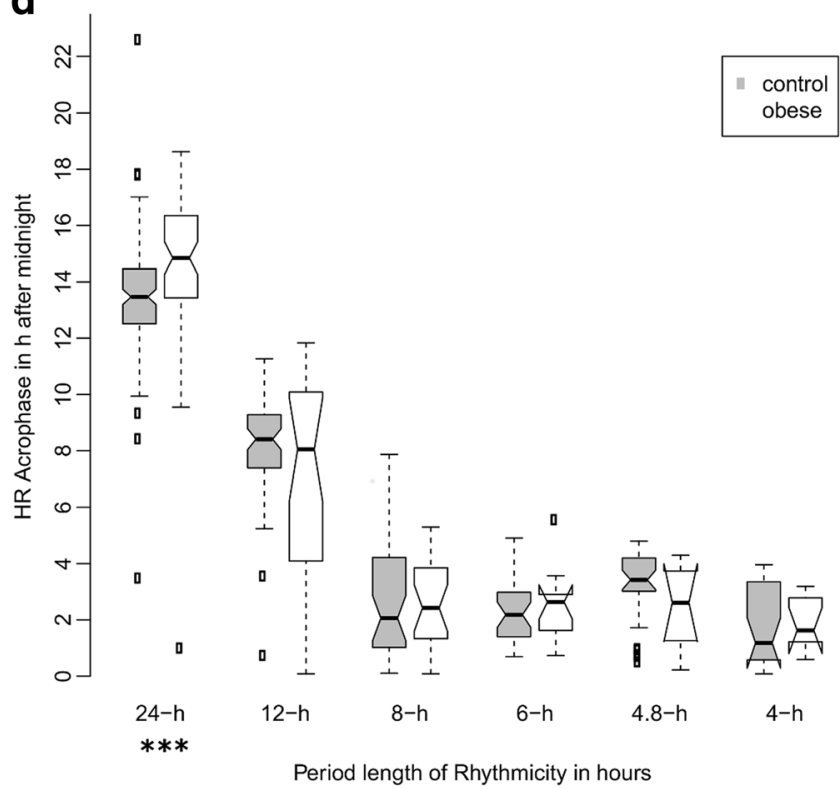

and ultradian rhythms. ${ }^{*} p<0.05$ using Mann-Whitney $U$ Test. b HR amplitudes of circadian and ultradian rhythms. ${ }^{* * *} p<0.001$ using Mann-Whitney $U$ Test. c MAP acrophases* of circadian and ultradian rhythms. ${ }^{* * *} p<0.001$ using Mann-Whitney $U$ Test. d HR acrophases of circadian and ultradian rhythms. ${ }^{* *} p<0.001$ using Mann-Whitney $U$ Test 
Table 2 Multivariate analysis for the prevalence of circadian and ultradian BP and HR rhythms in obese children and healthy children together

\begin{tabular}{lllll}
\hline Period length & $\begin{array}{l}\text { Significant } \\
\text { independent } \\
\text { variable }\end{array}$ & $\begin{array}{l}\text { Odds ratio } \\
(\text { OR })\end{array}$ & $\begin{array}{l}\text { OR confidence interval } \\
(2.5-97.5 \%)\end{array}$ & $p$ \\
\hline 24-h MAP & - & - & - & n.s. \\
12-h MAP & Age & 1.15 & $1.02-1.29$ & $<0.01$ \\
8-h MAP & - & - & - & n.s. \\
6-h MAP & - & - & - & n.s. \\
4.8-h MAP & - & - & - & n.s. \\
4-h MAP & Male sex & 0.35 & $0.16-0.76$ & $<0.01$ \\
& MAP 24 h SDS & 1.52 & $1.08-2.19$ & $<0.05$ \\
24-h HR & Age & 0.86 & $0.74-0.99$ & $<0.05$ \\
12-h HR & Age & 1.16 & $1.03-1.3$ & $<0.05$ \\
8-h HR & - & - & - & n.s. \\
6-h HR & Age & 1.22 & $1.06-1.42$ & $<0.01$ \\
4.8-h HR & - & - & - & n.s. \\
4-h HR & - & - & - & n.s. \\
\hline
\end{tabular}

Included variables are age (years), sex, height-SDS, BMI-SDS, 24-h MAP SDS, and 24-h heart rate (bpm) n.s. not significant

\section{Discussion}

The first finding of this study is that obese children show significantly altered circadian and ultradian rhythmicity. Thirty percent of the obese children were hypertensive. Second, the disturbance in circadian and ultradian rhythmicity for blood pressure and heart rate was independent of hypertensive BP levels and was therefore already present before hypertension became manifest.

Loss of nocturnal dipping, a recognized independent risk factor for cardiovascular disease [24], has been used as the only marker of circadian rhythmicity in the past. The present data confirm previous reports on the frequent lack of nocturnal dipping in obese children $[21,30]$.

Fourier analysis of 24-h blood pressure recordings allows a more detailed evaluation of blood pressure and heart rate rhythmicity. Normative values for children were already established 10 years ago [8]. Circadian and ultradian cardiovascular rhythms have been investigated in children with chronic renal failure (CRF) [41], in children with primary hypertension $(\mathrm{PH})$, and with white coat hypertension $(\mathrm{WCH})$ [17], as well as in children born small for gestational age (SGA) [40]. These reports show that there is an increasing perturbation of cardiovascular rhythms with increasing severity of the underlying disease. The obese participants showed only slight alterations of circadian and ultradian rhythms (reduced $24 \mathrm{~h}, 6$-h MAP prevalence, increased 4.8-h MAP amplitude, 24-h MAP acrophase, and 24-h HR acrophase and reduced $24 \mathrm{~h}$ HR amplitude), whereas children with CRF showed the highest number of alterations, followed by children with $\mathrm{PH}, \mathrm{WCH}$, and SGA. Consistent findings, which were also present in this study, were the loss of 24-h rhythmicity, the blunting of HR amplitudes, and the delaying of MAP and HR acrophases. In contrast, we found only a slight increase of the 4.8-h MAP amplitude.

The influence of age according to the multivariate analysis was comparable to the reference values of Hadtstein [8] with an increase in the prevalence of the 12-h MAP, the 12- and 6-h HR rhythms, and a decrease of the prevalence for the 24-h HR rhythm. In contrast, we could not show any influence of age on the 6-h MAP and 8-h MAP rhythm. In contrast to its potential influence on cardiovascular risk factors in childhood obesity [26], we only observed minor gender differences for cardiovascular rhythmicity parameters. Multivariate analysis for rhythm amplitudes and acrophases has demonstrated that BMI-SDS, age, height-SDS, and MAP-SDS all have an effect on amplitudes and acrophases. Disturbed cardiovascular rhythms correlated with visceral fat mass and elevated HR acrophases decreased after reduction of visceral fat [23]. Unfortunately, we are unable to assess the influence of visceral fat on cardiovascular rhythms because of incomplete data on waist circumference.

Taking into account that the perturbation of cardiovascular rhythms in this study was independent of hypertension and that previous studies $[17,40,41]$ showed increasingly disturbed cardiovascular rhythms with increasing severity of hypertension and underlying disease, one could argue whether disturbed cardiovascular rhythms could be the first sign of altered sympathetic regulation eventually leading to overt arterial hypertension [3].

Where circadian rhythms appear to be generated in the hypothalamic central pacemaker [27], ultradian cardiovascular rhythms depend more on sympathetic activity [3, 32]. Furthermore, hypertensive children might have increased 
sympathetic activity or decreased nocturnal parasympathetic activity $[14,31]$. Consistent with the disturbances in sympathetic regulation, the obese group in this study had a slightly, not significantly elevated 24 -h heart rate $(83.5$ vs. $86 \mathrm{bpm}$, $p=0.2$ ) but a significant elevation of heart rate during the nighttime (70.2 vs. 79 bpm, $p<0.001)$.

The alterations of rhythmicity in this study might be considered as an early effect of altered sympathetic activity comparable to alterations in the resting heart rate. The latter has been shown to be positively associated with blood pressure values [4] and with a clustering of cardiovascular risk factors in adolescents [6].

Other factors may also be responsible for disturbed cardiovascular rhythms in obese children. There is evidence that fragmented sleep, as seen with obstructive sleep apneas, results in loss of nocturnal dipping [19]. Likewise, the influence of age on rhythmicity parameters might be confounded by an altered sleep behavior of adolescents compared to younger counterparts. Furthermore, endocrine factors also seem to play a role. Lack of diurnal cortisol variations, for instance, as found in patients with central obesity, has been shown to be associated with loss of blood pressure dipping $[9,15]$. There is evidence that insulin resistance too is related to higher nocturnal blood pressure values in obese children [21]. Finally, adiponectin has been shown to be inversely correlated with ambulatory blood pressure parameters in obese adolescents [30].

The limitations of this study result from its retrospective design. There was no systematic recording of sleep behavior, particularly symptoms of obstructive sleep apneas. There was a lack of data of physical activity and missing data from the physical examination like incomplete collection of waist circumference and pubertal status. The latter might be of importance since circadian rhythms as well as ultradian rhythms have been shown to change during puberty [8]. On the other hand, the impact of this limitation might be reduced by having used an age- and gender-matched reference group. Furthermore, the influence of insulin resistance could not be assessed and finally, possible early target organ damage like an increase of intima-media thickness was not investigated.

In conclusion, this study demonstrates disturbed circadian and ultradian cardiovascular rhythms in obese children and adolescents, independently of hypertensive blood pressure levels. Fourier analysis of cardiovascular rhythms could be a valuable tool to detect early alterations of cardiovascular function eventually leading to hypertension. Thus, it would be of interest to prospectively assess whether there is a correlation between disturbed circadian and ultradian cardiovascular rhythms and target organ damage. This evidence provided, cardiovascular rhythmicity alterations assessed with 24$\mathrm{h}$ ABPM may become a reasonable, future diagnostic tool for early detection of obese children and adolescents at risk for hypertension.

Acknowledgments We thank Chantal Cripe-Mamie MD for her careful linguistic revision of this manuscript.

Author's contribution All the above listed authors contributed substantially to the conception of the study, the acquisition of the raw data and its analysis and interpretation. The initial manuscript has been consecutively edited for its scientific content and accuracy by all the listed authors and the revised version has been approved to be published.

\section{Compliance with ethical standards}

Ethical approval The study was approved by the local ethical review board and performed in accordance with the ethical standards as laid in the 1964 Declaration of Helsinki and its later amendments.

Informed consent All subjects and parents gave informed consent prior to inclusion in the study.

Conflict of interest The authors declare that they have no conflict of interest.

\section{References}

1. Aggoun Y, Farpour-Lambert NJ, Marchand LM, Golay E, Maggio $A B$, Beghetti M (2008) Impaired endothelial and smooth muscle functions and arterial stiffness appear before puberty in obese children and are associated with elevated ambulatory blood pressure. Eur Heart J 29:792-799

2. Braegger C, Jenni O, Konrad D, Molinari L (2011) Neue Wachstumskurven fuer die Schweiz. Paediatrica 22:10-11

3. Diedrich A, Jordan J, Tank J, Shannon JR, Robertson R, Luft FC, Robertson D, Biaggioni I (2003) The sympathetic nervous system in hypertension: assessment by blood pressure variability and ganglionic blockade. J Hypertens 21:1677-1686

4. Dong B, Wang Z, Wang HJ, Ma J (2015) The association between resting heart rate and blood pressure among children and adolescents with different waist circumferences. Eur J Pediatr 174:191197

5. Elkiran O, Yilmaz E, Koc M, Kamanli A, Ustundag B, Ilhan N (2013) The association between intima media thickness, central obesity and diastolic blood pressure in obese and overweight children: a cross-sectional school-based study. Int J Cardiol 165:528532

6. Farah BQ, Christofaro DGD, Balagopal PB, Cavalcante BR, de Barros MVG, Ritti-Dias RM (2015) Association between resting heart rate and cardiovascular risk factors in adolescents. Eur J Pediatr 174:1621-1628

7. Flynn JT, Daniels SR, Hayman LL, Maahs DM, McCrindle BW, Mitsnefes M, Zachariah JP, Urbina EM, American Heart Association Atherosclerosis H, Obesity in Youth Committee of the Council on Cardiovascular Disease in the Y (2014) Update: ambulatory blood pressure monitoring in children and adolescents: a scientific statement from the American Heart Association. Hypertension 63:1116-1135

8. Hadtstein C, Wuhl E, Soergel M, Witte K, Schaefer F, German Study Group for Pediatric H (2004) Normative values for circadian 
and ultradian cardiovascular rhythms in childhood. Hypertension 43:547-554

9. Holt-Lunstad J, Steffen PR (2007) Diurnal cortisol variation is associated with nocturnal blood pressure dipping. Psychosom Med 69:339-343

10. I'Allemand D, Wiegand S, Reinehr T, Muller J, Wabitsch M, Widhalm K, Holl R, Group AP-S (2008) Cardiovascular risk in 26,008 European overweight children as established by a multicenter database. Obesity (Silver Spring) 16:1672-1679

11. Jones A, Charakida M, Falaschetti E, Hingorani AD, Finer N, Masi S, Donald AE, Lawlor DA, Smith GD, Deanfield JE (2012) Adipose and height growth through childhood and blood pressure status in a large prospective cohort study. Hypertension 59:919-925

12. Juhola J, Magnussen CG, Viikari JS, Kahonen M, Hutri-Kahonen N, Jula A, Lehtimaki T, Akerblom HK, Pietikainen M, Laitinen T, Jokinen E, Taittonen L, Raitakari OT, Juonala M (2011) Tracking of serum lipid levels, blood pressure, and body mass index from childhood to adulthood: the Cardiovascular Risk in Young Finns Study. J Pediatr 159:584-590

13. Kimm SY, Obarzanek E (2002) Childhood obesity: a new pandemic of the new millennium. Pediatrics 110:1003-1007

14. Kohara K, Nishida W, Maguchi M, Hiwada K (1995) Autonomic nervous function in non-dipper essential hypertensive subjects. Evaluation by power spectral analysis of heart rate variability. Hypertension 26:808-814

15. Kumari M, Chandola T, Brunner E, Kivimaki M (2010) A nonlinear relationship of generalized and central obesity with diurnal cortisol secretion in the Whitehall II study. J Clin Endocrinol Metab 95: 4415-4423

16. Litwin M, Niemirska A, Sladowska J, Antoniewicz J, Daszkowska J, Wierzbicka A, Wawer ZT, Grenda R (2006) Left ventricular hypertrophy and arterial wall thickening in children with essential hypertension. Pediatr Nephrol 21:811-819

17. Litwin M, Simonetti GD, Niemirska A, Ruzicka M, Wuhl E, Schaefer F, Feber J (2010) Altered cardiovascular rhythmicity in children with white coat and ambulatory hypertension. Pediatr Res 67:419-423

18. Lobstein T, Frelut ML (2003) Prevalence of overweight among children in Europe. Obes Rev 4:195-200

19. Loredo JS, Nelesen R, Ancoli-Israel S, Dimsdale JE (2004) Sleep quality and blood pressure dipping in normal adults. Sleep 27: 1097-1103

20. Luma GB, Spiotta RT (2006) Hypertension in children and adolescents. Am Fam Physician 73:1558-1568

21. Lurbe E, Torro I, Aguilar F, Alvarez J, Alcon J, Pascual JM, Redon $\mathrm{J}$ (2008) Added impact of obesity and insulin resistance in nocturnal blood pressure elevation in children and adolescents. Hypertension 51:635-641

22. National High Blood Pressure Education Program Working Group on High Blood Pressure in C, Adolescents (2004) The fourth report on the diagnosis, evaluation, and treatment of high blood pressure in children and adolescents. Pediatrics 114:555-576

23. Niemirska A, Litwin M, Feber J, Jurkiewicz E (2013) Blood pressure rhythmicity and visceral fat in children with hypertension. Hypertension 62:782-788

24. Ohkubo T, Hozawa A, Yamaguchi J, Kikuya M, Ohmori K, Michimata M, Matsubara M, Hashimoto J, Hoshi H, Araki T, Tsuji I, Satoh H, Hisamichi S, Imai Y (2002) Prognostic significance of the nocturnal decline in blood pressure in individuals with and without high 24-h blood pressure: the Ohasama study. J Hypertens 20:2183-2189

25. Pall D, Juhasz M, Lengyel S, Molnar C, Paragh G, Fulesdi B, Katona E (2010) Assessment of target-organ damage in adolescent white-coat and sustained hypertensives. J Hypertens 28:2139-2144
26. Pires A, Martins P, Pereira AM, Marques M, Castela E, Sena C, Seica R (2016) Childhood adiposity: being male is a potential cardiovascular risk factor. Eur J Pediatr 175:63-69

27. Ralph MR, Foster RG, Davis FC, Menaker M (1990) Transplanted suprachiasmatic nucleus determines circadian period. Science 247: 975-978

28. Redwine KM, Acosta AA, Poffenbarger T, Portman RJ, Samuels J (2012) Development of hypertension in adolescents with pre-hypertension. J Pediatr 160:98-103

29. Richards J, Gumz ML (2013) Mechanism of the circadian clock in physiology. Am J Physiol Regul Integr Comp Physiol 304:R1053R1064

30. Shatat IF, Freeman KD, Vuguin PM, Dimartino-Nardi JR, Flynn JT (2009) Relationship between adiponectin and ambulatory blood pressure in obese adolescents. Pediatr Res 65:691-695

31. Sherwood A, Steffen PR, Blumenthal JA, Kuhn C, Hinderliter AL (2002) Nighttime blood pressure dipping: the role of the sympathetic nervous system. Am J Hypertens 15:111-118

32. Somers VK, Dyken ME, Mark AL, Abboud FM (1993) Sympathetic-nerve activity during sleep in normal subjects. N Engl J Med 328:303-307

33. Sorof JM, Cardwell G, Franco K, Portman RJ (2002) Ambulatory blood pressure and left ventricular mass index in hypertensive children. Hypertension 39:903-908

34. Stabouli S, Kotsis V, Papamichael C, Constantopoulos A, Zakopoulos N (2005) Adolescent obesity is associated with high ambulatory blood pressure and increased carotid intimal-medial thickness. J Pediatr 147:651-656

35. Steinberger J, Daniels SR, American Heart Association Atherosclerosis $\mathrm{H}$, Obesity in the Young $\mathrm{C}$, American Heart Association Diabetes C (2003) Obesity, insulin resistance, diabetes, and cardiovascular risk in children: an American Heart Association scientific statement from the Atherosclerosis, Hypertension, and Obesity in the Young Committee (Council on Cardiovascular Disease in the Young) and the Diabetes Committee (Council on Nutrition, Physical Activity, and Metabolism). Circulation 107: $1448-1453$

36. Suglia SF, Clark CJ, Gary-Webb TL (2013) Adolescent obesity, change in weight status, and hypertension: racial/ethnic variations. Hypertension 61:290-295

37. Tu W, Eckert GJ, DiMeglio LA, Yu Z, Jung J, Pratt JH (2011) Intensified effect of adiposity on blood pressure in overweight and obese children. Hypertension 58:818-824

38. Verdecchia P, Carini G, Circo A, Dovellini E, Giovannini E, Lombardo M, Solinas P, Gorini M, Maggioni AP, Group MS (2001) Left ventricular mass and cardiovascular morbidity in essential hypertension: the MAVI study. J Am Coll Cardiol 38:18291835

39. Wang Y, Lobstein T (2006) Worldwide trends in childhood overweight and obesity. Int J Pediatr Obes 1:11-25

40. Wolfenstetter A, Simonetti GD, Poschl J, Schaefer F, Wuhl E (2012) Altered cardiovascular rhythmicity in children born small for gestational age. Hypertension 60:865-870

41. Wuhl E, Hadtstein C, Mehls O, Schaefer F, Group ET (2005) Ultradian but not circadian blood pressure rhythms correlate with renal dysfunction in children with chronic renal failure. J Am Soc Nephrol 16:746-754

42. Wuhl E, Witte K, Soergel M, Mehls O, Schaefer F, German Working Group on Pediatric H (2002) Distribution of 24-h ambulatory blood pressure in children: normalized reference values and role of body dimensions. J Hypertens 20:1995-2007

43. Zuther SGaBL (2009) Chronos-Fit 1.06, http://www.ma.uniheidelberg.de/inst/phar/lehre/chrono.html. 